\title{
Difficultés diagnostiques de la maladie de Behçet chez l'enfant. Présentation pédiatrique et brève revue de la littérature.
}

\section{Difficult diagnosis of Behcet's disease. A pediatric case report and brief review of literature.}

\author{
Fettouma Mazari ${ }^{1}$, Karim Ait Idir ${ }^{2}$, Leila Boumati ${ }^{1}$
}

1. Service d'ophtalmologie CHU Hussein Dey Alger - Algérie

2. Service de pédiatrie. Centre de consultations spécialisées de

l’armée. Hussein Dey Alger Algérie

Correspondance à :

Karim AIT IDIR

aitidir22@gmail.com

DOI:https://doi.org/10.48087/B IMScr.2018.5126

Il s'agit d'un article en libre accès distribué selon les termes de la licence Creative Commons

Attribution International Licens (CC BY 4.0), qui autorise une utilisation, une distribution et une reproduction sans

restriction sur tout support ou format, à condition que l'auteur original et la revue soient

dûment crédités.

\section{RÉSUMÉ}

La maladie de Behçet (MB), décrite pour la première fois par le dermatologue turc Hulusi Behçet en 1937, est une vascularite occlusive généralisée chronique évoluant par poussée et rémission. Son diagnostic est purement clinique et est basé sur la présence obligatoire de certaines manifestations cliniques (aphtes buccaux, ulcérations génitales, uvéite, etc.). Les thromboses apparaissent comme une complication sévère et peuvent survenir tôt au cours de l'affection. La MB est rare chez l'enfant, caractérisée par un polymorphisme clinique et les critères obligatoires peuvent manquer au début de la maladie, entrainant un retard diagnostique. Nous rapportons le cas d'un adolescent de 10 ans dont le diagnostic de la MB n'a été confirmé que 2 ans après le début de sa maladie.

Mots-clés : Fièvre périodique, adolescent, thrombose cérébrale, uvéite, œdème papillaire, vascularite.

\section{ABSTRACT}

Behcet's disease (BD) is a chronic generalized vasculitis, described for the first time by the Turkish dermatologist Hulusi Behcet in 1937. It is characterized by a clinical presentation, based on the compulsory presence of unquestionable features like oral mouth ulcers, genital ulcerations and uveitis. Thromboses are severe complications and can occur early in the course of this affection. BD is rare in the child, with a clinical polymorphism, compulsory criteria may lack at the beginning of the disease, leading to a delay in diagnosis. We report the case of an 11-year child, whose diagnosis of DB was established 2 years after the beginning of symptoms.

Keywords: Recurrent fever, child, cerebral thrombosis, uveitis, papillary oedema, vasculitis.

\section{Introduction}

La maladie de Behçet (MB) est une cause rare d'uvéite chez l'enfant. Il s'agit d'une vascularite occlusive des petits et gros vaisseaux. Son diagnostic repose sur un faisceau d'arguments avec la présence d'aphtes buccaux récidivants, d'ulcères génitaux, d'une inflammation intraoculaire et de lésions cutanées. Le diagnostic est particulièrement difficile lors de formes incomplètes de la maladie, ce qui caractérise majoritairement les tableaux cliniques. L'hypopion est une manifestation rare, observé dans moins de $3 \%$ des cas [1]. Chez l'enfant, la forme la plus fréquente d'atteinte intraoculaire est une pan-uvéite associée à une importante rupture de la barrière hémato-oculaire [1,2]. Le risque majeur est celui de développer des complications rétiniennes ischémiques ou néovasculaires secondaires à une vascularite occlusive rétinienne. Les manifestations neurologiques inaugurales avec œdème papillaire bilatéral restent exceptionnelles chez l'enfant surtout si le tableau clinique initial est incomplet [3,4].

\section{Observation}

Un adolescent âgé de 11 ans, sans antécédents familiaux particuliers, dernier d'une fratrie de 3 enfants vivants bien portants et issu d'un couple non consanguin, a présenté en février 2015, soit à l'âge de 9 ans, des arthralgies de quatre grosses articulations et une fièvre $\left(39,5^{\circ} \mathrm{c}\right)$ éruptive avec une pustulose. Il a été traité en ambulatoire par des antibiotiques et des anti-inflammatoires, qui ont amélioré temporairement son état (disparition de la fièvre, diminution de l'intensité des arthralgies).

Après une courte période, et toujours en 2015, il y'a eu une reprise des arthralgies associées à un œdème articulaire, et un autre épisode fébrile avec érythème noueux d'où son hospitalisation, durant laquelle l'exploration n'a rien objectivé de particulier (FNS, Bilan inflammatoire, bilan d'hémostase, bilan hépatique et rénal, Immunoélectrophorèse des protéines, ASIO, test de Mantoux, et à deux reprises le Facteur anti-nucléaire et enzyme de conversion de l'angiotensine et le bilan urinaire). Cependant, il a été noté pour la première fois, une aphtose buccale minime. Devant la négativité du bilan biologique, il a été décidé encore une fois une antibiothérapie (association amoxicilline, gentamicine) et un traitement anti-inflammatoire non stéroïdien au long cours (type acide acétyle salicylique).

Une rémission a été obtenue jusqu'en janvier 2016 (âge de 10 ans) où une nouvelle récidive du même tableau clinique a été notée, accompagnée d'une baisse de l'acuité visuelle, de vertige et de céphalées rebelles, d'où son orientation vers le service d'ophtalmologie. L'examen spécialisé a retrouvé une baisse de l'acuité visuelle à $7 / 10$ à droite et à $6 / 10$ à gauche, une uvéite antérieure non granulomateuse modérée sans synéchies postérieures, et au fond de l'œil un œè̀me papillaire bilatéral franc stade 2 d'allure inflammatoire (figure 1) associé à quelques vascularites périphériques bilatérales confirmées à l'angiographie (figure 2). Le champ visuel de Goldman a retrouvé aussi un rétrécissement des isoptères périphériques modérés (figure 3). L'examen des potentiels visuels évoqués était sans particularités. Devant les céphalées intenses et l'œdème papillaire bilatéral, une angio-IRM orbito-cérébrale a été pratiquée objectivant une thrombose veineuse de la partie postérieure du sinus sagittal (figure 4). Par ailleurs, nous avons pratiqué un supplément d'exploration, n'ayant rien trouvé d'anormal (fibrinogène, anti thrombine, protéine $\mathrm{C}$ et $\mathrm{S}$, anticorps anti-phospholipides, anticorps et facteur anti nucléaires, homcystinémie, sérologies infectieuses (HIV, EBV, TPHA, VRDL, CMV, toxoplasmose), Antigène HLA B51) ainsi que l'étude du liquide céphalo-rachidien.

Quelques mois plus tard, une aggravation de l'aphtose buccale avec des récidives fréquentes a été notée. Ainsi, le diagnostic de MB ou plutôt un neuro-Behcet a été retenu avec : la négativité du bilan infectieux, l'apparition d'une aphtose buccale, les antécédents d'érythème noueux, et d'arthralgies, l'uvéo-papillite, les signes neurologiques, la vascularite rétinienne et la thrombose vasculaire cérébrale. 

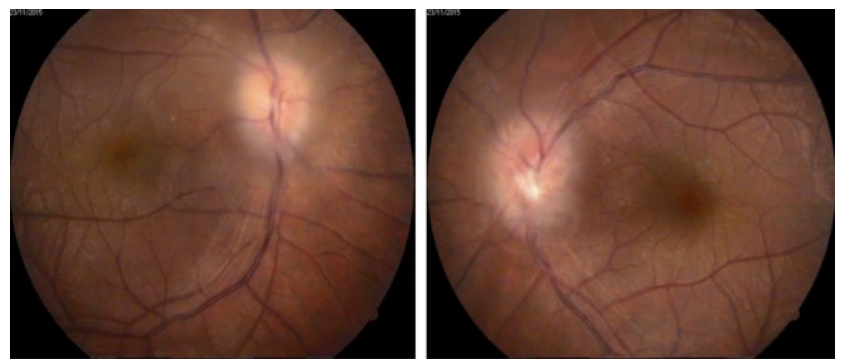

Figure 1. Rétinographie du fond d'œil met en évidence un œdème papillaire bilatéral stade II.
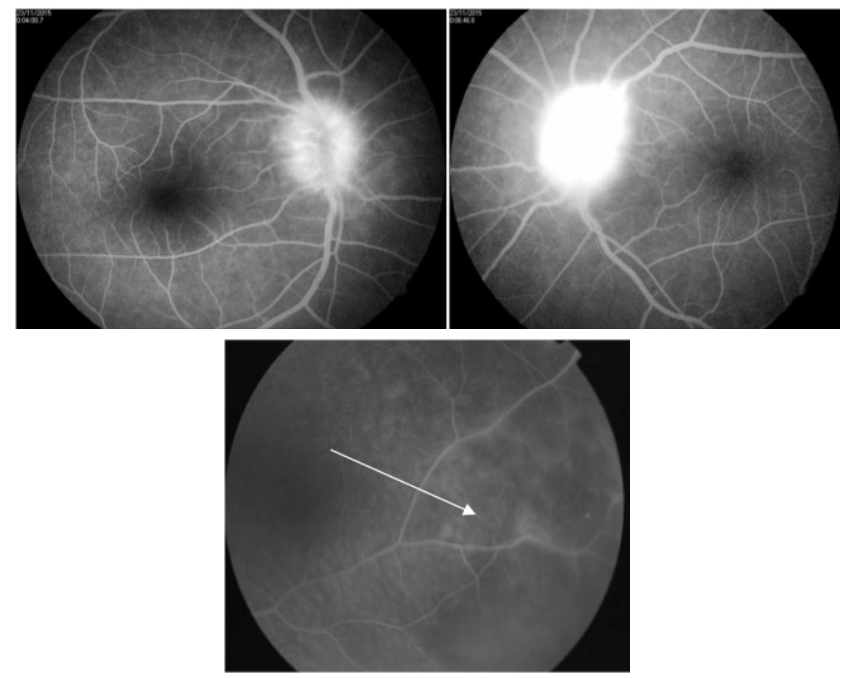

Figure 2. œdème papillaire bilatéral avec discrète vascularite périphérique (flèche blanche).

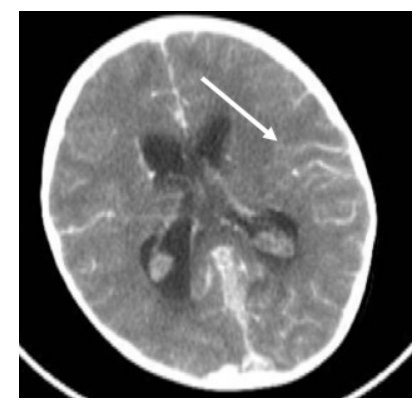

Figure 4. Thrombose veineuse de la partie postérieure du sinus sagittal (flèche blanche).

Figure 3. Champs visuel de Goldman retrouvant un rétrécissement des isoptères périphériques aux deux yeux.

pédiatre, de l'ophtalmologiste, du radiologue avec surveillance stricte et régulière des manifestations oculaires et neurologiques.

\section{Discussion}

La MB est une vascularite habituellement observée chez l'adulte jeune. Les formes pédiatriques sont rares puisqu'une centaine de cas seulement ont été rapportés dans la littérature [5]. Ceci serait dû à la difficulté diagnostique en l'absence des signes cardinaux de la MB au début de son évolution. Sa fréquence varie de $0,9 \%$ à 7,6 \% selon les séries avec des particularités pédiatriques cliniques et évolutives différentes de celles de l'adulte [6]. Son pronostic demeure sombre et conditionné par l'atteinte oculaire particulièrement sévère dans cette tranche d'âge. La MB se caractérise par des épisodes récurrents d'aphtose buccale et/ou génitale avec ulcérations, accompagnées de manifestations systémiques [7] :

$\checkmark$ Cutanées: nodules hypodermiques (érythème noueux), pseudo folliculite nécrotique, réaction anormale aux traumatismes cutanés (pathergie),

$\checkmark$ Oculaires : uvéite antérieure et postérieure très agressive (risque de cécité),

$\checkmark$ Articulaires : arthrite des grosses articulations,

$\checkmark$ Digestives : lésions ulcéreuses au niveau du tube digestif,

$\checkmark$ Vasculaires: thromboses veineuses, anévrismes artériels (notamment au niveau des artères pulmonaires), Budd Chiari, péricardite, myocardite,

$\checkmark$ Neurologiques : méningite ou méningo-encéphalite aseptique avec ou sans hypertension intracrânienne,

Le diagnostic de MB chez l'enfant est difficile à poser et il n'est pas rare que le délai entre le début des symptômes et le diagnostic soit de plusieurs mois ou années (cas de notre patient (presque 2 ans). Il est également important de souligner que plus l'enfant est jeune, plus il sera difficile d'affirmer un diagnostic, ceci étant dû au problème de l'expression de la douleur et à la verbalisation de celle-ci. La fièvre est un symptôme commun en pédiatrie et dans la majorité des cas elle est d'origine infectieuse. Chez le petit enfant, la fréquence des épisodes infectieux de la sphère ORL et des voies respiratoires font que le médecin se retrouve alors confronté à des épisodes fébriles récidivants, pour lesquels des pathologies auto-inflammatoires doivent être considérées dans le diagnostic différentiel. Le syndrome de PFAPA (Periodic Fever, Aphtous stomatitis, Pharyngitis, cervical Adenitis) est la plus fréquente de ces maladies, excepté dans les régions où la Fièvre méditerranéenne familiale (FMF) est prévalente. Chez notre patient la fièvre était placée au premier plan et le recours aux antibiotiques malgré l'absence de foyers infectieux était systématique. 


\section{CAS CLINIQUE}

Les manifestations articulaires sont relativement fréquentes avec l'atteinte cutanéo-muqueuse et oculaire. Elles peuvent être inaugurales ou revêtir des aspects atypiques pouvant errer le diagnostic. Elles surviennent dans 45 à $76 \%$ des cas selon les séries [8]. Parfois, elles précédent l'aphtose de plusieurs mois, voire de plusieurs années (cas de notre patient avec presque 2 ans), ou êtres concomitants à l'aphtose ou apparaitre après celle-ci $[9,10]$.

Il semblerait que l'atteinte oculaire soit aussi fréquente chez l'enfant que chez l'adulte [11] mais chez les moins de 10 ans elle est plus rare [12]. L'âge moyen du début de l'uvéite est de 14 ans avec un délai moyen entre 2 et 3 ans avant la déclaration de la maladie et selon les séries, il y'a une légère prédominance masculine, et l'atteinte est souvent bilatérale [13]. Le risque majeur est celui de développer des complications rétiniennes ischémiques ou néovasculaires secondaires à une vasculite rétinienne occlusive. Par ailleurs, le pronostic visuel est menacé par la survenue de cataracte, d'œdème maculaire, et d'atrophie optique [14].

Les thromboses veineuses sont rares chez l'enfant. Elles sont estimées à 6,6 \% [15]. La maladie peut se présenter (mais rarement) sous forme d'un œdème papillaire bilatéral secondaire à une thrombose cérébrale (cas de notre patient). L'atteinte neurologique peut correspondre à une méningite, à une méningo-encéphalite ou à une thrombose veineuse cérébrale. Plusieurs cas associent MB et hypertension intracrânienne, comme dans notre observation, l'enfant présentait des céphalées, vertiges avec œdème papillaire bilatéral et une discrète baisse de l'acuité visuelle.

La MB avec vascularite(s) est une maladie piégeuse chez l'enfant qui peut s'accompagner d'une fièvre intermittente sans cycle particulier. Les aphtes sont traditionnellement bucco-génitaux et sont considérés comme un critère obligatoire ; cependant, leur apparition peut être retardée jusqu'à la puberté ce qui peut faire errer le diagnostic pendant plusieurs mois voire plusieurs années comme le cas échéant.

Il n'y a aucun test de laboratoire spécifique de la MB et le diagnostic de cette affection est clinique. Actuellement, chez l'adulte, on se réfère aux critères internationaux révisés de l'International Study Group for Behcet's Disease (ISGBD) [16-17]. A l'opposé, en pédiatrie, plusieurs auteurs considèrent que ces critères ne sont pas applicables vu la rareté de l'atteinte oculaire, de la fréquence des manifestations cliniques inhabituelles et la difficulté à différencier la MB d'autres affections, surtout inflammatoires iléales [17]. En pratique courante, et on considérant un patient donné, il faut rester critique vis-à-vis de ces critères surtout chez l'enfant.

\section{Conclusion}

La MB est une entité rare en pédiatrie. Sa fréquence n'est pas bien établie chez l'enfant, cela est dû aux différentes tranches d'âge, l'origine ethnique et géographique et surtout à l'absence de critères diagnostiques unifiés. Les signes oculaires sont de mauvais pronostic et peuvent apparaitre à n'importe quel stade de la maladie. La prise en charge est multidisciplinaire entre ophtalmologiste, pédiatre, radiologue et immunologiste.

Déclaration d'intérêts : les auteurs ne déclarent aucun conflit d'intérêt en rapport avec cet article.

\section{Références}

1. Wentworth BA, Freitas-Neto CA, Foster CS. Management of pediatric uveitis. F1000Prime Reports. 2014 ; 6:41. doi: 10.12703/P6-41.

2. Guex-Crosier Y et al. Prise en charge d'une uvéite pédiatrique .Paediatrica. Vol. 26 No. 5 .2015.

3. Guex-Crosier et al. L'uvéite non infectieuse de l'enfant. Recommandations des groupes de travail de l'Uvéite et de Rhumatologie pédiatrique. Forum Médical Suisse 2012 ; 38(12) : 739-41.

4. Clarke LA et al. Epidemiology of uveitis in children over a 10-year period. Clin Exp Rheumatol 2013 ; 31(4): 633-7.

5. Laghmari A.et al. La maladie de Behçet chez l'enfant, aspects cliniques et évolutifs A propos de 13 cas .Journal français d'ophtalmologie. Journal Français d'Ophtalmologie Vol 25, $\mathrm{N}^{\circ} 9$ - novembre 2002. pp. 904-908

6. Atmani S et al. La maladie de Behçet chez l’enfant : à propos de trois cas pédiatriques. Journal de pédiatrie et de puériculture 2006 ; $19: 313-317$.

7. Bhalerao PM et al. A case of Behcet's disease posted for surgery: anaesthetic implications. Ind Journal of Anaesthesiology 2015; 59: 517-9.

8. Turki S, Turki S, Ben Taarit C, Ben Matz H. La maladie de Behçet : une série de 400 cas. Rev Med Interne 2002; 23(1):67.

9. Ben Taarit C, Turki S, Ben Maiz H. Les manifestations rhumatologiques de la maladie de Behçet: à propos de 309 cas. Rev Med Interne 2001 ; $22: 1049-55$.

10. Khammar Z et al. Les manifestations articulaires au cours de la maladie de Behçet. A propos de 60 cas. Revue du Rhumatisme 2007, doi:10.1016/j.rhum.2007.10.592.

11. Atmaca L et al. Behcet disease in children. Ocul Immunol Inflamm $2011 ; 19: 103-7$.

12. Sungur GK et al Clinical and demographic evaluation of Behçet disease among different paediatric age groups British Journal of Ophthalmology 2009 ; $93: 83-87$.

13. M. Piram, I. Koné-Paut .La Maladie de Behcet de l'enfant La Revue de médecine interne 35 (2014) 121-125.

14. Chaker .N et al. Manifestations oculaires au cours de la maladie de Behcet chez l'enfant. Journal de pédiatrie et de puériculture (2013) 26, 257-260.

15. Kone-Paut I, Bernard JI. La maladie de Behçet chez l'enfant en France. Archives Françaises de pédiatrie. 1993; 50:561-5.

16. Davatchi F, Schirmer M, Zouboulis CC, et al. Evaluation and revision of the International Study Group Criteria for Behçet's disease. ACR $2007:$ Abstr 465.

17. Chebbia W et al. Maladie de Behcet juvénile : à propos de quatre observations. Journal de pédiatrie et de puériculture (2013) $26,326-331$. 